\title{
Characteristics and outcomes of elderly patients with diffuse gliomas: a multi-institutional cohort study by Kansai Molecular Diagnosis Network for CNS Tumors
}

\author{
Takahiro Sasaki $^{1,2} \cdot$ Junya Fukai $^{1,2}$ ([) $\cdot$ Yoshinori Kodama ${ }^{2,3,18} \cdot$ Takanori Hirose $^{2,4} \cdot$ Yoshiko Okita $^{2,5}$. \\ Shusuke Moriuchi ${ }^{2,6} \cdot$ Masahiro Nonaka $^{2,7} \cdot$ Naohiro Tsuyuguchi $^{2,8} \cdot$ Yuzo Terakawa $^{2,8} \cdot$ Takehiro Uda $^{2,8}$.

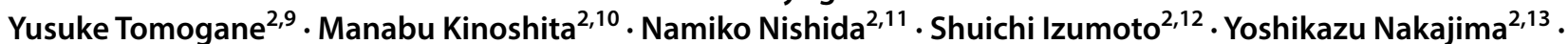

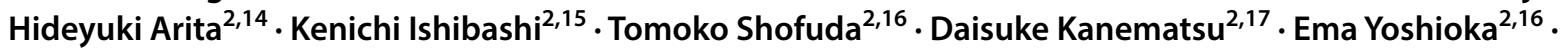 \\ Masayuki Mano $^{2,18} \cdot$ Koji Fujita $^{1,2} \cdot$ Yuji Uematsu ${ }^{1,2} \cdot$ Naoyuki Nakao $^{1,2} \cdot$ Kanji Mori $^{2,19} \cdot$ Yonehiro Kanemura $^{2,5,17}$
}

Received: 21 April 2018 / Accepted: 11 July 2018 / Published online: 3 August 2018

(c) The Author(s) 2018

\begin{abstract}
Introduction This study investigates the current state of clinical practice and molecular analysis for elderly patients with diffuse gliomas and aims to elucidate treatment outcomes and prognostic factors of patients with glioblastomas.

Methods We collected elderly cases ( $\geq 70$ years) diagnosed with primary diffuse gliomas and enrolled in Kansai Molecular Diagnosis Network for CNS Tumors. Clinical and pathological characteristics were analyzed retrospectively. Various factors were evaluated in univariate and multivariate models to examine their effects on overall survival.

Results Included in the study were 140 elderly patients (WHO grade II: 7, III: 19, IV: 114), median age was 75 years. Sixtyseven patients (47.9\%) had preoperative Karnofsky Performance Status score of $\geq 80$. All patients underwent resection (grosstotal: $20.0 \%$, subtotal: $14.3 \%$, partial: $39.3 \%$, biopsy: $26.4 \%)$. Ninety-six of the patients (68.6\%) received adjuvant treatment consisting of radiotherapy (RT) with temozolomide (TMZ). Seventy-eight of the patients (75.0\%) received radiation dose of $\geq 50$ Gy. MGMT promoter was methylated in 68 tumors (48.6\%), IDH1/2 was wild-type in 129 tumors (92.1\%), and TERT promoter was mutated in 78 of 128 tumors (60.9\%). Median progression-free and overall survival of grade IV cases was 8.2 and 13.6 months, respectively. Higher age ( $\geq 80$ years) and TERT promoter mutated were associated with shorter survival. Resection and adjuvant RT + TMZ were identified as independent factors for good prognosis.

Conclusions This community-based study reveals characteristics and outcomes of elderly glioma patients in a real-world setting. Elderly patients have several potential factors for poor prognosis, but resection followed by RT + TMZ could lengthen duration of survival.
\end{abstract}

Keywords Elderly $\cdot$ Diffuse glioma $\cdot$ Glioblastoma $\cdot$ Molecular marker $\cdot$ Prognostic factor $\cdot$ Real-world data

Takahiro Sasaki and Junya Fukai have contributed equally to this work.

Electronic supplementary material The online version of this article (https://doi.org/10.1007/s11060-018-2957-7) contains supplementary material, which is available to authorized users.

Junya Fukai

junfukai@wakayama-med.ac.jp

Extended author information available on the last page of the article

\section{Introduction}

Diffuse gliomas are the most common primary central nervous system tumors, accounting for about $30 \%$ of all brain tumors in Japan [1]. Median age at glioblastoma (WHO grade IV) diagnosis is 63.0 years and higher than median age at diagnosis of lower-grade gliomas [1]. Recently, the number and percentage of elderly people are rising in Japan, and hence diffuse gliomas in the elderly are becoming more common $[1,2]$. As this demographic tends to fare worse than non-elderly population, there are major concerns regarding prediction of clinical behavior and treatment outcomes. 
In clinical practice, physicians are usually apprehensive in offering aggressive treatments to elderly patients because of concerns relating to treatment tolerance due to advanced age, co-morbidities or underlying propensity for complications $[3,4]$. Although treatment-associated toxicity in the elderly appears to be higher and optimal treatment for elderly patients remains controversial, treatment tolerance seems to be dependent on individual predisposition as well as comorbid conditions [5-8]. Clinical consequences are often complicated by additional considerations common to elderly populations.

Adult diffuse gliomas have highly variable clinical behavior, response to therapy, and outcomes $[9,10]$. Recently, mutations in IDH, TP53, TERT promoter and codeletion of chromosome arms $1 \mathrm{p}$ and $19 \mathrm{q}(1 \mathrm{p} / 19 \mathrm{q}$ codeletion) have been highlighted as clinically relevant prognostic markers of diffuse gliomas [9-13]. Some of these molecular parameters are required for integrative diagnosis for 2016 CNS WHO Classification [14]. Some molecular markers have also been reported as being predictive of the potential benefit from specific therapeutic intervention. Particularly in elderly patients, $M G M T$ promoter methylation status is reportedly to be important information for deciding adjuvant treatment regimen $[8,15-17]$. The prevalence and impact of previously established biomarkers are considered as a main area of investigation for diffuse gliomas in the elderly.

This study aims to demonstrate the current state of clinical practice for elderly patients with diffuse gliomas and molecular analyses of diffuse gliomas in the elderly. In the multi-institutional retrospective cohort study of 140 elderly cases treated at 13 hospitals in Kansai Molecular Diagnosis Network for CNS Tumors (Kansai Network), we elucidate both clinical and pathological features of elderly glioma cases, as well as treatment outcomes and prognostic factors of glioblastoma (GB) patients in a real-world setting.

\section{Methods}

\section{Ethics}

This study was carried out in accordance with the principles of the Helsinki declaration. Approval was obtained from the Institutional Review Board of Wakayama Medical University (No. 98), Osaka National Hospital (No. 713), and all collaborative institutes. Written informed consent was obtained from all patients.

\section{Patient population}

This study included patients ( $\geq 70$ years) who were treated at 13 institutions or hospitals participating in the Kansai Network. Established in the Kansai area of Western Japan, the
Kansai Network collects tumor samples and clinical information from affiliated hospitals and analyzes molecular status of tumors for diagnosis and research. Between September 2007 and September 2016, we collected total 918 samples including all kinds of primary and recurrent gliomas. From this data bank, we focused on primary diffuse gliomas of the elderly and collected elderly cases (Online Resource 1). Each institution provided between two and 20 patients. Diagnosis of diffuse gliomas was initially confirmed by histopathological examination at each institution or hospital (Online Resource 1).

\section{Clinical information}

Clinical information was collected from medical records including patient demographics, preoperative KPS scores, extent of surgical resection (EOR), adjuvant radiation and chemotherapy (RCT) regimens, and survival time. EOR was classified as gross total resection (GTR, $>95 \%$ of the tumor was resected), subtotal resection (STR, 90-94\%), partial resection (PR, $<90 \%)$ and biopsy according to the assessment by the surgeon. Patients received RCT consisting of radiation (RT) plus concomitant and adjuvant temozolomide (TMZ), RT alone, TMZ monotherapy or none [18]. Adjuvant RCT regimens were determined by attending physicians considering the patient's condition.

\section{Histopathological examination}

All cases underwent central pathology review by senior board-certified neuropathologists (Y.K. and T.H.). Integrated diagnosis and WHO grading were made based on the 2016 WHO Classification of Tumors of the CNS (2016 WHO) [14].

\section{Genetic analysis}

Tumor DNA was extracted using a DNeasy Blood \& Tissue Kit (Qiagen, Tokyo, Japan). Details of genetic analysis, including PCR and sequencing for each gene status, were previously reported [19]. The presence of hotspot mutations in IDHI (R132) and IDH2 (R172) was assessed by Sanger sequencing in all cases [19]. The two mutation hotspots in the TERT promoter were also analyzed by Sanger sequencing [12]. The copy number status of $1 p-19 q$ was determined by multiplex ligation-dependent probe amplification (MLPA) (Oligodendroglioma 1p-19q probemix and EK1 reagent kit, MRC-Holland, Amsterdam, Netherlands). The methylation status of the MGMT promoter was analyzed by quantitative methylation specific PCR (qMSP) after bisulfite modification of genomic DNA [20]. Based on an outcome-based study to determine an optimal cutoff to judge $M G M T$ promoter methylation in a series of newly diagnosed 
glioblastomas (GB), we used a cut-off of $\geq 1 \%$ for $M G M T$ methylation.

\section{Statistical analysis}

Statistical analysis was performed using an SAS package and JMP Pro version 12 (SAS Institute, Cary, NC, USA). Categorized data were compared between subgroups using Chi square test. Overall survival curves were estimated by Kaplan-Meier method and compared with log-rank test. Univariate and multivariate analyses of risk factors were performed using Cox proportional hazards model. A $\mathrm{P}$-value $<0.05$ was considered statistically significant.

\section{Results}

\section{Clinical characteristics}

Table 1 shows clinical characteristics of the 140 patients analyzed in this study. There were 78 males (55.7\%) and 62 females (44.3\%) with a median age of 75 years (range 70-93 years). Based on integrated diagnosis of 2016 WHO Classification, 114 patients $(81.4 \%)$ were grade IV, 19 (13.6\%) were III, and 7 (5.0\%) were II [14]. Preoperative KPS scores ranged from 30 to 100 (median 70), and 67 patients (47.9\%) had a score of $\geq 80$. Regarding EOR, 28 patients (20.0\%), 20 (14.3\%), 55 (39.3\%), and 37 (26.4\%) underwent GTR, STR, PR, and biopsy, respectively. After resection, 96 patients (68.6\%), 24 (17.1\%), and 8 (5.7\%) received combined RT and TMZ (RT+ TMZ), TMZ monotherapy and RT alone, respectively.

Sixty-six patients (47.1\%) were admitted within 2 years from the time of analysis (Online Resources 1,2). Observation period ranged from 0.7 to 48.5 months (median 10.6 months). Tumor progression was observed in 79 patients (56.4\%). 67 patients (47.9\%) were deceased at the time of analysis. Median progression-free survival (mPFS) was 8.4 months ( 8.2 months in grade IV), and median overall survival (mOS) was 14.7 months (13.6 months in grade IV).

Table 2 shows treatment regimen according to age, preoperative KPS score and WHO grade. Distribution of EOR, especially tumor reduction versus biopsy, was not significantly different among groups in age, preoperative KPS score and WHO grade. Regardless of higher age ( $\geq 80$ years) and lower KPS score ( $<70$ years), maximum and safe resection was intended to perform. Adjuvant treatment regimen was not significantly associated with preoperative KPS score but with both age and WHO grade. Patients ( $\geq 80$ years) were significantly less likely to receive radiation $(\mathrm{p}<0.0001)$.

\section{Molecular characteristics}

Table 1 also shows molecular characteristics and frequencies of each genetic status. $M G M T$ promoter methylation was present in 68 tumors (48.6\%). IDH1/2 mutation was detected in only 11 tumors (7.7\%). Notably, IDHI/2 was wild-type in 4 of 7 grade II gliomas (57.1\%) and 13 of 18 grade III (72.2\%) (Online Resource 3). 1p/19q co-deletion was seen in 7 of 59 tumors (11.9\%). TERT promoter mutations were observed in 84 of 138 tumors $(60.9 \%)$, including 7 oligodendroglial tumors $(7 / 8,87.5 \%)$ and 69 GB $(69 / 113$, $61.1 \%$ ) (Online Resource 3).

Results of integrated diagnosis according to the 2016 WHO Classification are shown in Table 1. Almost all grade IV gliomas were GB, IDH-wildtype (98.2\%). Grade II and III astrocytomas consisted of $16 \mathrm{IDH}$-wildtype (94.1\%) and $1 \mathrm{IDH}$-mutant (5.9\%). In nine oligodendroglial tumors, two anaplastic oligodendroglioma (AO) lacked both IDH mutation and $1 \mathrm{p} / 19 \mathrm{q}$ codeletion.

Arita et al. proposed molecular classification based on $I D H$ and TERT mutation, which divided into four molecular groups, each showing distinct patient characteristics, histology, or clinical outcome [12]. The combined IDH/TERT classification is shown in Online Resource 3. There were a small number in $I D H$ mutant (IDHmut) groups (10/138, $7.2 \%$ ) regardless of TERT mutation status. The group with mutations in both $I D H$ and TERT consisted of only oligodendroglial tumors $(6 / 6,100 \%)$. The group with mutation in IDH but not TERT contained two anaplastic astrocytoma and two GB. In $I D H$ wild-type (IDHwt) groups, there were a larger number of tumors harboring TERT mutation (75/128, $58.6 \%)$ than those without mutation. In these groups, GB was the most common $(45 / 53,84.9 \%$ and $66 / 75,88 \%)$.

\section{Treatment outcomes and prognostic factors}

Table 3 and Fig. 1 show median survival times and Kaplan-Meier survival curves of WHO grade IV cases, respectively. Table 3 also includes the results of univariate analyses of the relationships between factors and PFS/ OS. Patients ( $\geq 80$ years) had significantly shorter OS (9.5 months) compared to 14.6 months in those $<80$ years old ( $\mathrm{p}=0.0068)$ (Fig. 1a and Online Resource 4a). Patients with preoperative KPS score of $<70$ tended to survive for a shorter time than those with KPS 80-100 (10.5 versus 16.2 months, $\mathrm{p}=0.1700$ ) (Fig. 1b). The group that underwent aggressive surgical resection (GTR + STR) had significantly longer OS (19.1 months) than those who received biopsy (10 months) $(p=0.0104)$ (Fig. 1c). Notably, the resection group had longer OS than those who received non-aggressive treatment ( $\mathrm{PR}+$ biopsy) (13 months) and this difference was also significant $(\mathrm{p}=0.0310)($ Online Resource $4 \mathrm{~b})$. 
Table 1 Clinical and molecular characteristics of the cohort

\begin{tabular}{|c|c|c|}
\hline Characteristic $(n=140)$ & Data & $(\%)$ \\
\hline \multicolumn{3}{|l|}{ Age (years) } \\
\hline Median (range) & \multicolumn{2}{|l|}{$75(70-93)$} \\
\hline $70-74$ & 62 & $(44.3)$ \\
\hline $75-79$ & 41 & (29.3) \\
\hline $80-$ & 37 & (26.4) \\
\hline \multicolumn{3}{|l|}{ Gender } \\
\hline Male & 78 & $(55.7)$ \\
\hline Female & 62 & $(44.3)$ \\
\hline \multicolumn{3}{|l|}{ WHO grade } \\
\hline Grade II & 7 & $(5.0)$ \\
\hline Grade III & 19 & (13.6) \\
\hline Grade IV & 114 & (81.4) \\
\hline \multicolumn{3}{|l|}{ Preoperative KPS score } \\
\hline $80-100$ & 67 & $(47.9)$ \\
\hline-70 & 72 & (51.4) \\
\hline Unknown & 1 & $(0.7)$ \\
\hline \multicolumn{3}{|l|}{ Extent of surgical resection } \\
\hline GTR & 28 & (20.0) \\
\hline STR & 20 & (14.3) \\
\hline PR & 55 & $(39.3)$ \\
\hline Biopsy & 37 & (26.4) \\
\hline \multicolumn{3}{|l|}{ Adjuvant treatment } \\
\hline $\mathrm{RT}+\mathrm{TMZ}$ & 96 & (68.6) \\
\hline TMZ monotherapy & 24 & (17.1) \\
\hline RT alone & 8 & (5.7) \\
\hline None & 12 & (8.6) \\
\hline \multicolumn{3}{|l|}{ Radiation dose (Gy) } \\
\hline $50-60$ & 78 & $(75.0)$ \\
\hline$<50$ & 20 & $(19.2)$ \\
\hline Unknown & 6 & (5.8) \\
\hline \multicolumn{3}{|l|}{ Genetic status } \\
\hline \multicolumn{3}{|l|}{$M G M T$ promoter } \\
\hline Methylated & 68 & $(48.6)$ \\
\hline Unmethylated & 72 & $(51.4)$ \\
\hline \multicolumn{3}{|l|}{$I D H 1 / 2$} \\
\hline Wild type & 129 & $(92.1)$ \\
\hline Mutant & 11 & (7.7) \\
\hline$T E R T$ promoter mutation & $84 / 138$ & $(60.9)$ \\
\hline $1 \mathrm{p} / 19 \mathrm{q}$ codeletion & $7 / 59$ & (11.9) \\
\hline TP53 mutation & $50 / 140$ & $(35.7)$ \\
\hline \multicolumn{3}{|l|}{2016 WHO classification } \\
\hline Diffuse astrocytoma, IDH-wildtype & 4 & \\
\hline Anaplastic astrocytoma, IDH-mutant & 1 & \\
\hline Anaplastic astrocytoma, IDH-wildtype & 12 & \\
\hline Glioblastoma, IDH-wildtype & 112 & \\
\hline Gliosarcoma & 1 & \\
\hline Glioblastoma, IDH-mutant & 1 & \\
\hline Oligodendroglioma, IDH-mutant and $1 \mathrm{p} / 19 \mathrm{q}$-codeleted & 3 & \\
\hline $\begin{array}{l}\text { Anaplastic oligodendroglioma, IDH-mutant and } \\
1 \mathrm{p} / 19 \mathrm{q} \text {-codeleted }\end{array}$ & 4 & \\
\hline $\begin{array}{l}\text { Anaplastic oligodendroglioma, NOS (lacking IDH- } \\
\text { mutation and } 1 \mathrm{p} / 19 \mathrm{q} \text {-codeletion) }\end{array}$ & 2 & \\
\hline
\end{tabular}

WHO World Health Organization, KPS Karnofsky Performance Status
Patients who received adjuvant $\mathrm{RT}+\mathrm{TMZ}$ had $\mathrm{mOS}$ of 16.2 months, while mOS of those who received TMZ monotherapy or RT alone was 11 or 7.5 months. RT + TMZ group had significantly longer OS than others $(\mathrm{p}=0.0007)$ (Fig. 1d).

MGMT promoter methylated group showed better PFS compared to unmethylated group (9.5 months compared to 7 months) $(\mathrm{p}=0.0129)$, whereas no significant difference was found in OS between them (13.7 months vs. 10 months) $(p=0.3240)$ (Online Resource 5a). Between these groups, there was no significant difference in distribution of treatment regimen (Online Resource 6). Based on MGMT methylation status, estimated survival times according to adjuvant treatments were compared (Fig. 1e, f). RT + TMZ group had a significantly longer OS time than other groups in both MGMT methylated patients (20.1 months) $(\mathrm{p}=0.0005)$ and in unmethylated patients (13.8 months) $(\mathrm{p}=0.0083)$. Although the number of RT group $(n=5$, $\mathrm{mOS}=11.1$ months) was too small for statistical analyses, significant difference was found between RT + TMZ and RT groups in MGMT unmethylated patients. TMZ group had a significantly shorter OS time than RT + TMZ group even in methylated groups $(\mathrm{n}=14, \mathrm{mOS}=12.9$ months $)$.

Regarding TERT mutation status, there was a significant difference between wild-type and mutated groups (Table 3 and Online Resource 5b). TERTmut group had significantly shorter OS (10.4 months) than TERTwt group (18 months) $(\mathrm{p}=0.0109)$. Association between TERT mutation and MGMT methylation was investigated (Online Resource 6 and Fig. 1g). As the result, the interaction between TERT and $M G M T$ was significant (PFS, $\mathrm{p}=0.0074$; OS, $\mathrm{p}=0.0071$ ) and TERTmut/MGMTunmet group had the poorest prognosis (8.8 months), followed by TERTmut/MGMTmet group (13.6 months).

In the WHO grade IV cohort, there was only one case with $I D H$ mutation. Despite the small number even in WHO grade II-IV cases, IDHmut group had significantly longer survival ( $\mathrm{mPFS}=27.2$ months, $\mathrm{mOS}=29.7$ months) than IDHwt group (mPFS $=8.2$ months, $\mathrm{mOS}=13.8$ months $)$ (PFS, $\mathrm{p}=0.0003$; OS, $\mathrm{p}=0.0175$ ) (Online Resource 5c). Based on the IDH/TERT classification, Kaplan-Meier survival curves of the four groups are shown in Fig. 1h. There appeared to be four distinct curves $(p=0.0109)$, but the observation period was too short for conclusive statistical results in the IDHmut groups. Among the IDHwt groups, there was a significant difference in OS between TERTwt and TERTmut groups $(\mathrm{p}=0.0316)$ and IDHwt/TERTmut group had the shortest OS (11.8 months).

For WHO grade II-IV cases, univariate and multivariate analyses identified WHO grade as an independent prognostic factor (Online Resources 7, 8). As the results of univariate analysis of the relationships between characteristics and estimated survival times for WHO grade IV 
Table 2 Treatment regimen according to age, preoperative KPS score and WHO grade

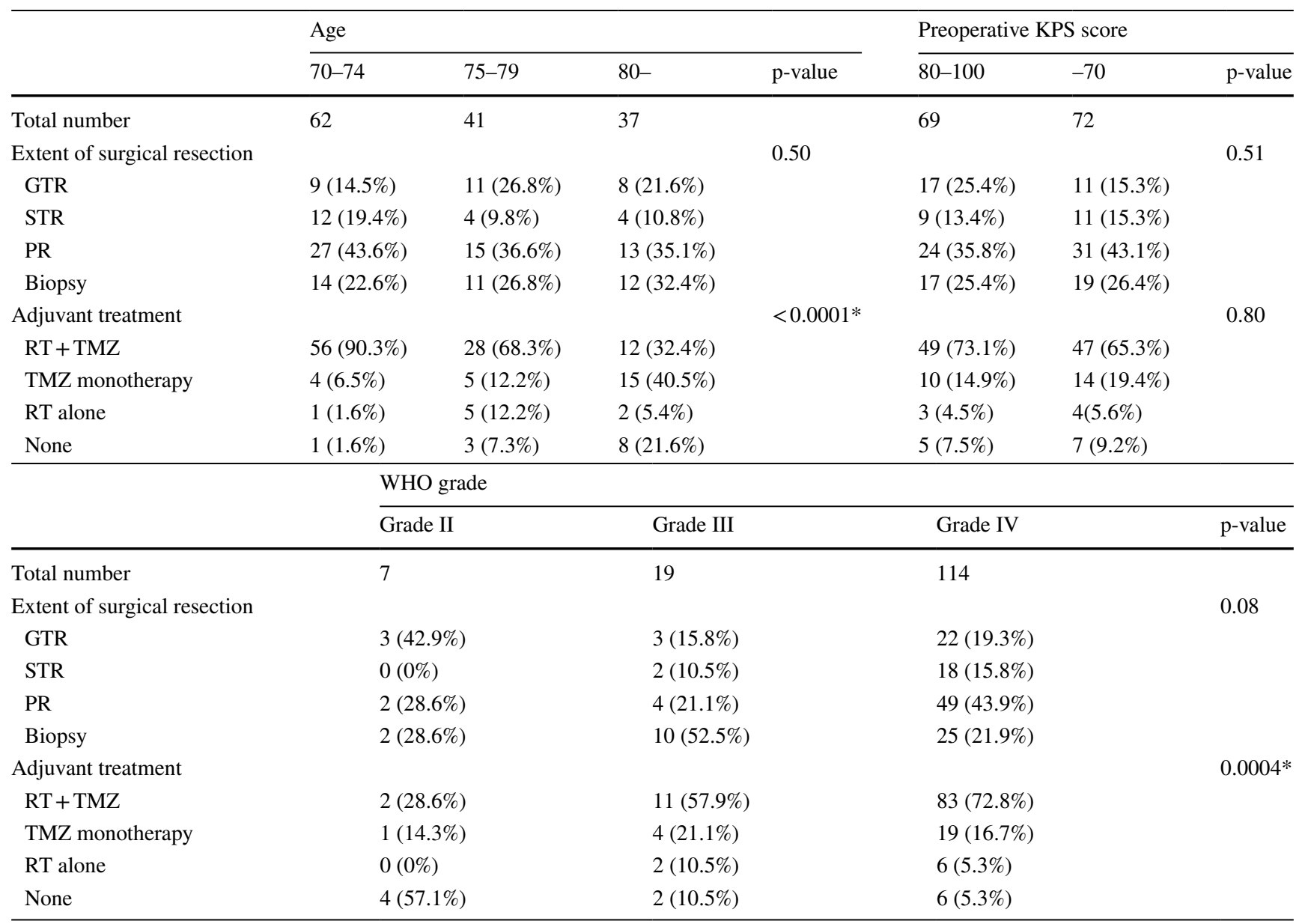

*Pearson's Chi square test was applied for the statistical analysis. $\mathrm{p}<0.05$, significant difference

cases, age, resection, adjuvant treatment and TERT mutation status were significantly associated with longer OS (Table 3; Fig. 1 and Online Resource 4). Table 4 shows the results of multivariate analysis of factors associated with OS. Resection and adjuvant treatment (RT + TMZ) were identified as independent factors for good prognosis.

\section{Discussion}

In this study, we retrospectively analyzed 140 elderly patients with diffuse gliomas treated at 13 hospitals in the Kansai Network. The cohort had several clinical and molecular characteristics: age $\geq 80$ years $(26.4 \%)$, lowergrade glioma (18.6\%), preoperative KPS scores below 70 (51.4\%), resection (34.3\%), adjuvant RT + TMZ (68.6\%), MGMT promoter methylation (48.6\%), IDH1/2 mutation (7.7\%) and TERT promoter mutation (60.9\%). Higher age ( $\geq 80$ years) and TERT promoter mutated were associated with poor prognosis. Resection and adjuvant RT $+\mathrm{TMZ}$ were identified as independent good prognostic factors.

Large cohort studies of elderly patients with diffuse gliomas are limited [4]. This study included 140 patients with an age of $\geq 70$ years. Statistical analyses reconfirms previous reports that age is one of the most important prognostic factors and performance status is independently associated with survival $[4,21]$.

As the result according to 2016 WHO Classification, several characteristics were notable. First, grade II/III astrocytomas consisted of IDH-wt (94.1\%) and IDH-mut $(5.9 \%)$. Generally, the great majority falls into the IDHmut category and IDH-wt astrocytomas are uncommon [22]. Moreover, in oligodendroglial tumors, some AO did not retain both $I D H$ mutations and $1 \mathrm{p} / 19 \mathrm{q}$ codeletion (22\%). These tumors, histologically typical oligodendrogliomas, were diagnosed as AO, NOS after careful evaluation.

Arita et al. reported that almost all tumors harboring concurrent IDHI/2 mutations and total 1p/19q loss had TERT 
Table 3 Results of univariate analyses of the relationship between factors and survivals in WHO Grade IV cases

\begin{tabular}{|c|c|c|c|c|}
\hline Factors & $\begin{array}{l}\text { Median progression-free } \\
\text { survival times (months) }\end{array}$ & p-value & $\begin{array}{l}\text { Median overall sur- } \\
\text { vival times (months) }\end{array}$ & p-value \\
\hline WHO grade IV & & & 13.6 & \\
\hline Age & & 0.0824 & & $0.0068 *$ \\
\hline $70-79$ & 8.5 & & 14.6 & \\
\hline $80-$ & 7.2 & & 9.5 & \\
\hline Preoperative KPS score & & 0.3517 & & 0.1700 \\
\hline $80-100$ & 8.2 & & 16.2 & \\
\hline $0-70$ & 8.3 & & 10.5 & \\
\hline Extent of surgical resection & & 0.3068 & & $0.0104 *$ \\
\hline Resection & 8.4 & & 19.1 & \\
\hline Biopsy & 8 & & 10 & \\
\hline Adjuvant treatment & & 0.1184 & & $0.0007 *$ \\
\hline $\mathrm{RT}+\mathrm{TMZ}$ & 8.6 & & 16.2 & \\
\hline TMZ monotherapy & 8.1 & & 11 & \\
\hline RT alone & 3.5 & & 7.5 & \\
\hline Radiation dose & & 0.2878 & & 0.3837 \\
\hline $50-$ & 8.6 & & 16.2 & \\
\hline-50 & 6.5 & & 13.8 & \\
\hline$M G M T$ promoter & & $0.0129 *$ & & 0.3240 \\
\hline Methylated & 9.5 & & 13.7 & \\
\hline Unmethylated & 7 & & 13 & \\
\hline$T E R T$ promoter & & 0.6869 & & $0.0109 *$ \\
\hline Wild type & 8.4 & & 18 & \\
\hline Mutated & 7.9 & & 10.4 & \\
\hline
\end{tabular}

$*<0.05$, significant difference promoter mutations [23]. Indeed, in TERT mutated tumors, all tumors with $I D H$ mutation harbored $1 \mathrm{p} / 19 \mathrm{q}$ codeletion. Moreover, they proposed combined IDH/TERT classification [12]. Also in this study, IDHwt/TERTmut patients expected the shortest survival in four subgroups.

Wiestler et al. reported that $M G M T$ promoter methylation was $35 \%$ in malignant astrocytoma in the elderly ( $>65$ years) [24]. Our cohort had a relatively high frequency of $M G M T$ methylation (48.6\%). Grade IV patients with MGMT methylated tumors showed better survival compared to those with unmethylated tumors, but the difference did not reach statistical significance. This trend was also observed in previous reports $[4,8]$.

TERT mutation status had a prognostic impact in this study. Together with MGMT methylation status, significant interaction between TERT and MGMT was observed. GB patients with TERT mutated and MGMT unmethylated had the poorest prognosis. Based on the results, a combination of $I D H, T E R T$, and $M G M T$ would refine clinically relevant classification of elderly diffuse gliomas.

Several treatment options have been recommended [16]. For low-grade gliomas in the elderly, adjuvant treatments have never been discussed. In the cohort, non-aggressive resection tended to be undertaken. On the other hand, adjuvant RT + TMZ was conducted in the majority. Notably, there was no significant difference in survival time between 50 and $60 \mathrm{~Gy}$ and $<50 \mathrm{~Gy}$ RT groups, which is discussed in several studies [6, 8, 25, 26]. TMZ monotherapy group resulted in shorter survival regardless of $M G M T$ status, as was inconsistent with other reports [17].

TMZ concomitant with and adjuvant to RT is a widely used approach, but the role in elderly cases remains discussed [27]. Arvold et al. stated that the addition of TMZ to RT was associated with a small survival gain [28]. Franceschi et al. reported that RT $+\mathrm{TMZ}$ is effective only in methylated MGMT tumors [29]. On the other hand, in the phase 3 trial by Perry et al., the addition of TMZ to short-course RT was associated with significantly longer survival [3]. In subgroup analyses, the benefit was also observed in unmethylated MGMT cases [3]. These results suggest that the addition of TMZ to RT confers a survival benefit regardless of $M G M T$ status.

In general, treatment outcomes are mostly consistent with previous reports $[4,21,30]$. Although the optimal treatment remains controversial, maximum and safe resection followed by short course RT with concurrent and adjuvant TMZ is warranted in GB [3]. 
a Age

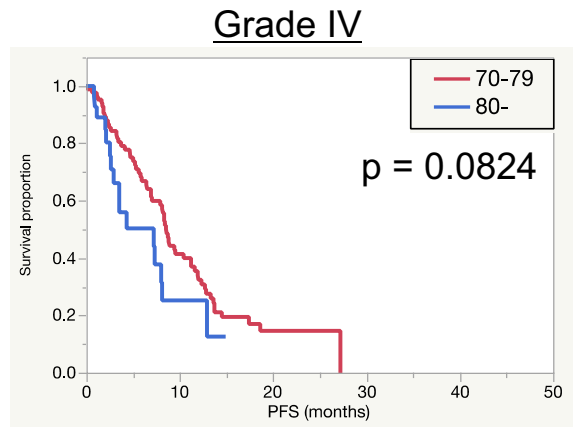

Number at risk

$\begin{array}{lllll}70-79 & 84 & 30 & 5 & 0\end{array}$

80- $\quad 28 \quad 5$

\section{b Preoperative KPS score}

\section{Grade IV}

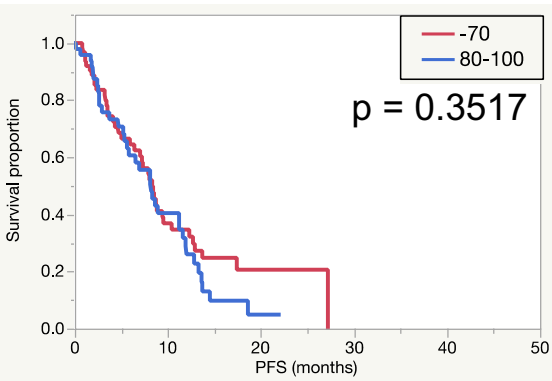

Number at risk

$\begin{array}{llll}-70 & 63 & 18 & 4\end{array}$

80-100

\section{c Extent of surgical resection}

Grade IV

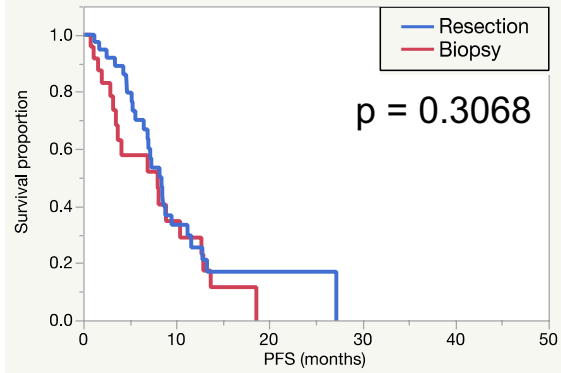

Number at risk

$\begin{array}{lllll}\text { Resectio } & 39 & 11 & 2 & 0\end{array}$

n

Biopsy

Fig. 1 Kaplan-Meier survival curves according to age (a), preoperative KPS score (b), extent of surgical removal (c) and adjuvant treatment (d), adjuvant treatments in MGMT promotor methylated (e)

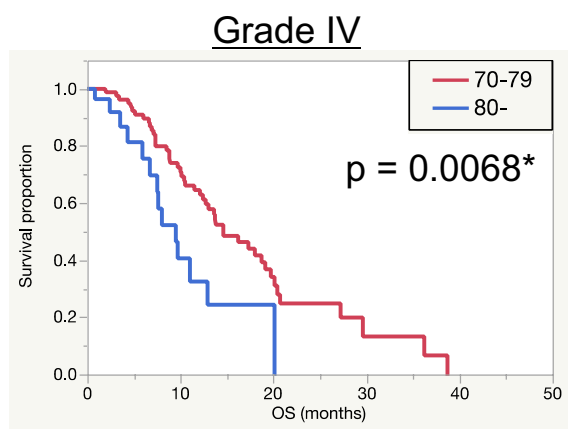

Number at risk

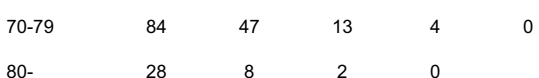

$80-$

\section{Grade IV}

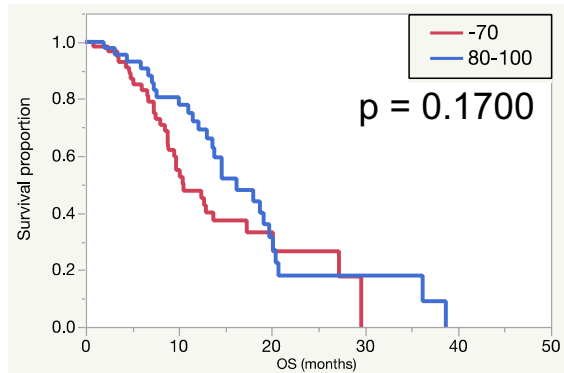

Number at risk

$\begin{array}{lllll}-70 & 63 & 25 & 7 & 0\end{array}$

$\begin{array}{llllll}80-100 & 48 & 30 & 8 & 3 & 0\end{array}$

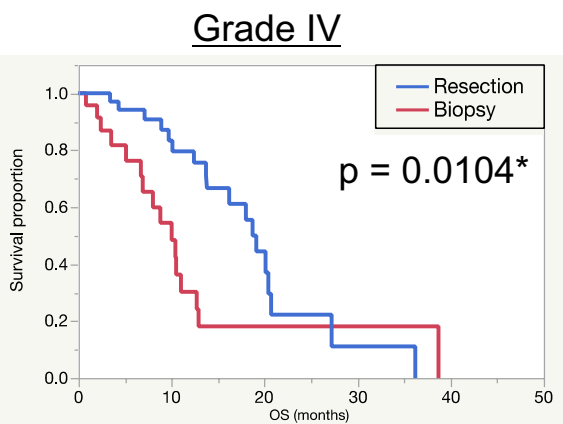

Number at risk

$\begin{array}{llllll}\text { Resection } & 39 & 23 & 7 & 2 & 0\end{array}$

$\begin{array}{llllll}\text { Biopsy } & 24 & 9 & 2 & 2 & 0\end{array}$

or unmethylated (f) patients, TERT/MGMT interaction (g) in WHO Grade IV cases and IDH/TERT classification in WHO Grade II-IV cases (h) 


\section{d Adjuvant treatment}

Grade IV

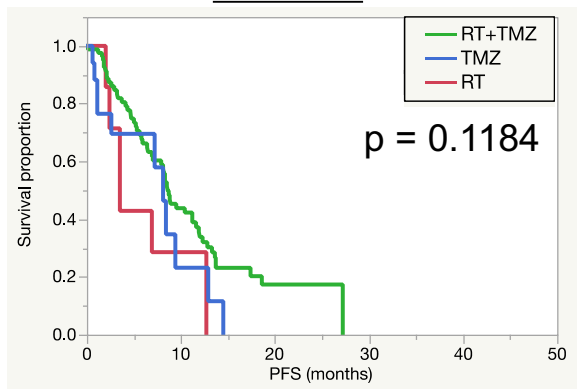

Number at risk

$\begin{array}{lllll}\mathrm{RT}+\mathrm{TMZ} & 82 & 30 & 5 & 0\end{array}$

$\begin{array}{llll}\text { TMZ } & 17 & 3 & 0\end{array}$

$\begin{array}{llll}\text { RT } & 7 & 3 & 0\end{array}$

e MGMT methylated

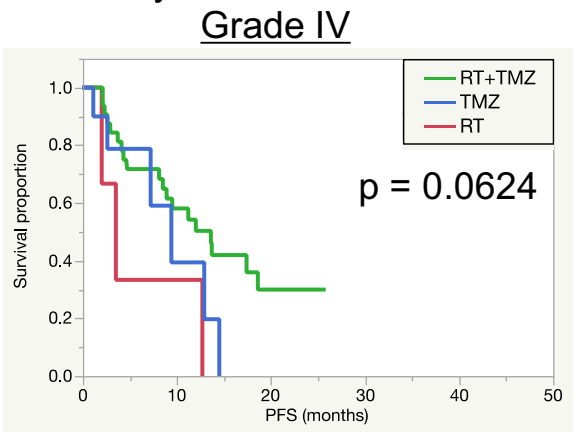

Number at risk

$\begin{array}{lccc}\text { RT }+ \text { TMZ } & 34 & 18 & 4 \\ \text { TMZ } & 10 & 3 & 0 \\ \text { RT } & 3 & 2 & 0\end{array}$

f MGMT unmethylated

\section{Grade IV}

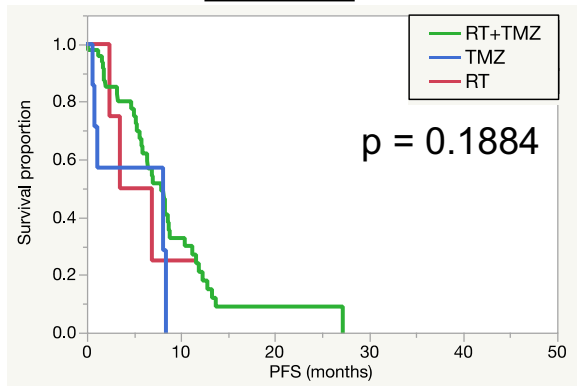

Number at risk

$\begin{array}{lllll}\text { RT+TMZ } & 48 & 13 & 2 & 0\end{array}$

$\begin{array}{llll}\text { TMZ } & 7 & 1 & 0\end{array}$

RT

Fig. 1 (continued)

\section{Grade IV}

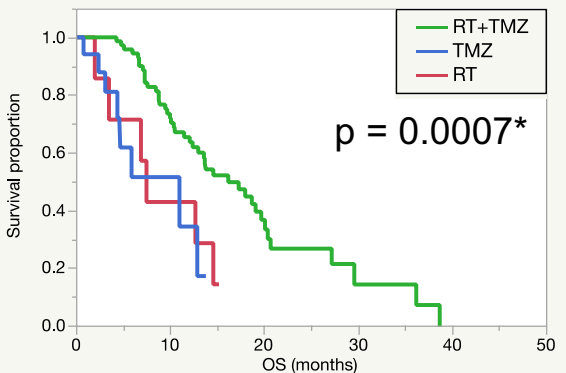

Number at risk

$\begin{array}{llllll}\text { RT+TMZ } & 82 & 46 & 12 & 3 & 0\end{array}$

$\begin{array}{lll}\text { TMZ } & 17 & 4\end{array}$

$\begin{array}{lll}\text { RT } & 7 & 4\end{array}$

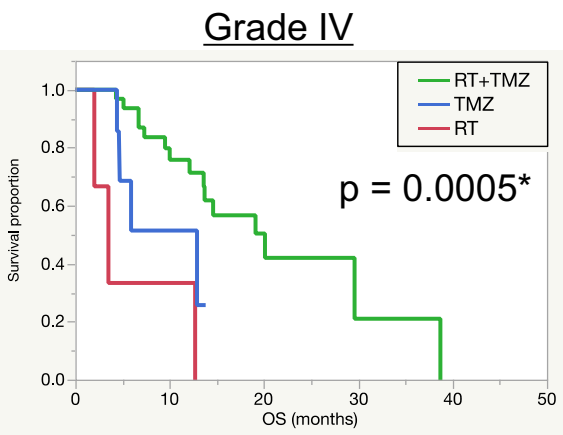

Number at risk

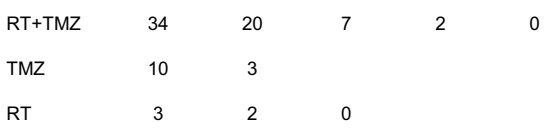




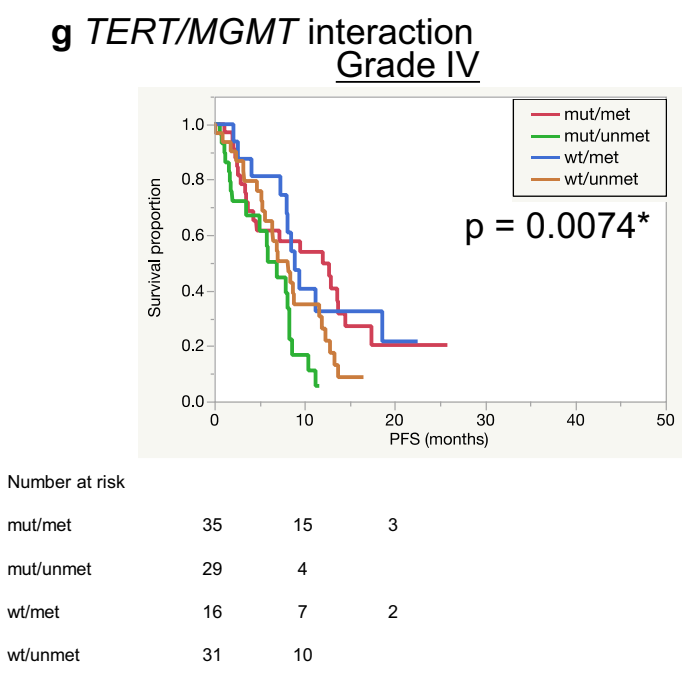

h IDHITERT classification
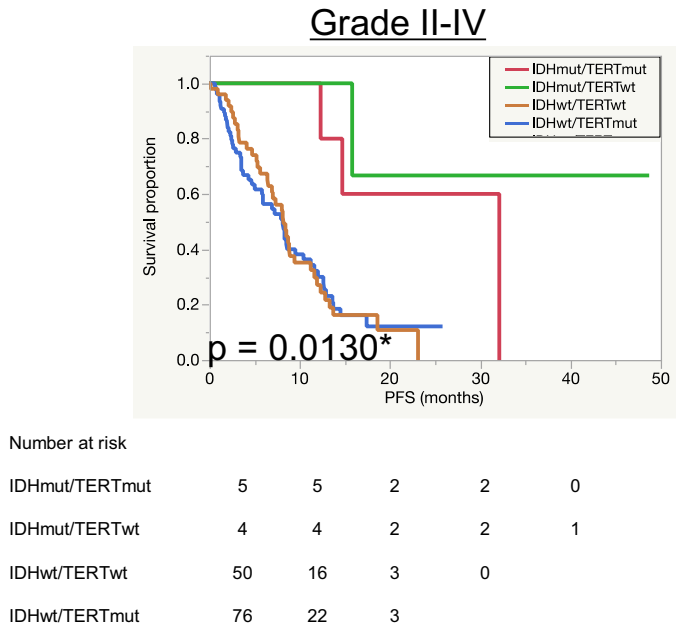

Number at risk

IDHmut/TERTmut

IDHmut/TERTwt

IDHwt/TERTwt
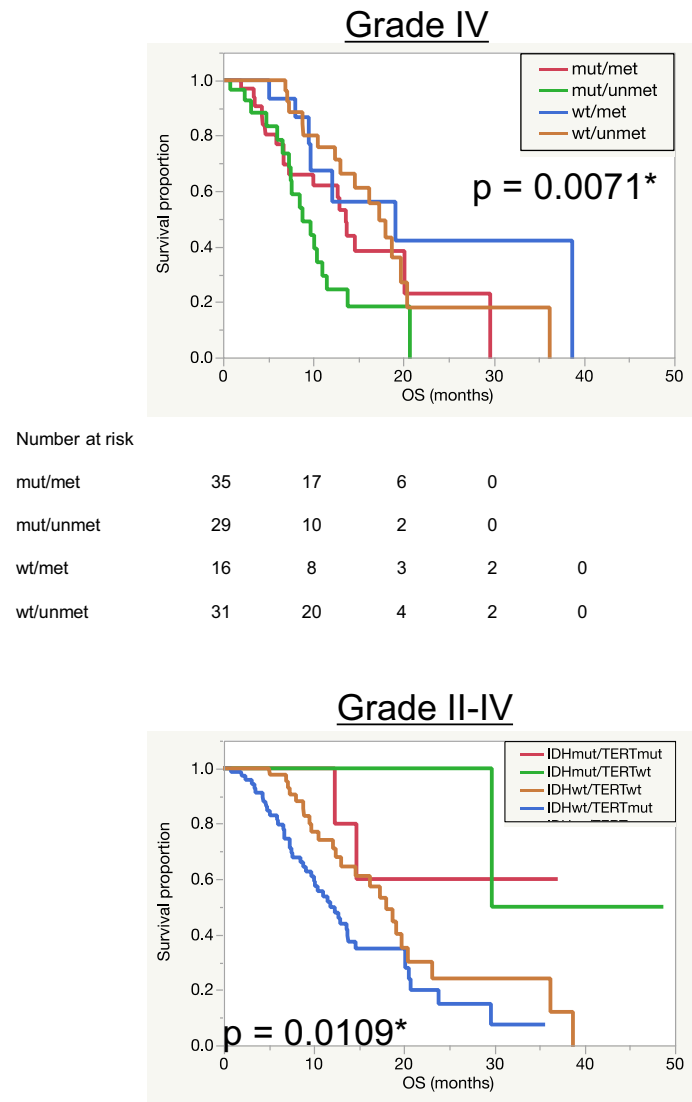

Fig. 1 (continued)

Table 4 Results of multivariate analyses of factors associated with overall survival in WHO Grade IV cases

\begin{tabular}{lll}
\hline Factors & Hazard ratio $(95 \% \mathrm{Cl})$ & $\mathrm{p}$-value \\
\hline Age $(<80)$ & $0.6128(0.3776-1.815)$ & 0.8161 \\
$\begin{array}{l}\text { Extent of surgical resection } \\
\quad \text { (resection) }\end{array}$ & $0.5052(0.2726-0.8903)$ & $0.0175^{*}$ \\
Adjuvant treatment (RT + TMZ) & $0.4105(0.2032-0.8701)$ & $0.0209^{*}$ \\
TERT promoter (wild-type) & $0.5890(0.3345-1.013)$ & 0.0559 \\
\hline
\end{tabular}

$* \mathrm{p}<0.05$, significant difference

As a multi-institutional retrospective cohort design, there are several limitations. Unlike a randomized study, selection bias on decision-making of treatment strategy could exist. Attending physicians may decide to deliver treatments considering the patients' age, conditions and wishes, and thus a selection could affect the survival findings. Variation of treatment regimen at multiple institutions, such as radiation protocol and dose schedule, should also be considered. The limited number of patients could explain the absence of statistical power to detect differences between groups. Modest prognostic impact of molecular characteristics might be partly due to the limited follow-up period of the population.

In conclusion, we report characteristics and outcomes of elderly patients with diffuse gliomas in the Kansai Network. This community-based study elucidated the present status of real-world practice. Further investigation in a larger population would contribute to our better understanding of the pathogenesis of glioma in the elderly.

Acknowledgements We thank Ms. Ai Takada at the Institute for Clinical Research, Osaka National Hospital, National Hospital Organization and Ms. Motoko Namiki at the Department of Neurological Surgery, Wakayama Medical University for their excellent assistance. 


\section{Compliance with ethical standards}

Conflict of interest The authors declare that they have no conflict of interest.

Ethical approval All procedures performed in studies involving human participants were in accordance with the ethical standards of the institutional and/or national research committee and with the 1964 Helsinki declaration and its later amendments or comparable ethical standards.

Informed consent Informed consent was obtained from all individual participants included in the study.

Open Access This article is distributed under the terms of the Creative Commons Attribution 4.0 International License (http://creativeco mmons.org/licenses/by/4.0/), which permits unrestricted use, distribution, and reproduction in any medium, provided you give appropriate credit to the original author(s) and the source, provide a link to the Creative Commons license, and indicate if changes were made.

\section{References}

1. Committee of Brain Tumor Registry of Japan (2017) Report of Brain Tumor Registry of Japan (2005-2008). Neurol Med Chir (Tokyo) 57(suppl-1):1-102

2. Committee of Brain Tumor Registry of Japan (2014) Report of Brain Tumor Registry of Japan (2001-2004). Neurol Med Chir (Tokyo) 54(suppl.):1-102

3. Perry JR, Laperriere N, O'Callaghan CJ et al (2017) Short-course radiation plus temozolomide in elderly patients with glioblastoma. N Engl J Med 376:1027-1037

4. Álvarez de Eulate-Beramendi S, Álvarez-Vega MA, Balbin M et al (2016) Prognostic factors and survival study in high-grade glioma in the elderly. Br J Neurosurg 30:330-336

5. Jordan JT, Gerstner ER, Batchelor TT, Cahill DP, Plotkin SR (2016) Glioblastoma care in the elderly. Cancer 122:189-197

6. Zarnett OJ, Sahgal A, Gosio J et al (2015) Treatment of elderly patients with glioblastoma: a systematic evidence-based analysis. JAMA Neurol 72:589-596

7. Arvold ND, Reardon DA (2014) Treatment options and outcomes for glioblastoma in the elderly patient. Clin Interv Aging 9:357-367

8. Wick W, Platten M, Meisner C et al (2012) Temozolomide chemotherapy alone versus radiotherapy alone for malignant astrocytoma in the elderly: the NOA-08 randomised, phase 3 trial. Lancet Oncol 13:707-715

9. The Cancer Genome Atlas Research Network (2015) Comprehensive, integrative genomic analysis of diffuse lower-grade gliomas. N Engl J Med 372:2481-2498

10. Eckel-Passow JE, Lachance DH, Molinaro AM et al (2015) Glioma groups based on $1 \mathrm{p} / 19 \mathrm{q}, I D H$, and TERT promoter mutations in tumors. N Engl J Med 372:2499-2508

11. Suzuki H, Aoki K, Chiba K et al (2015) Mutational landscape and clonal architecture in grade II and III gliomas. Nat Genet 47:458-468

12. Arita H, Yamasaki K, Matsushita Y et al (2016) A combination of TERT promoter mutation and MGMT methylation status predicts clinically relevant subgroups of newly diagnosed glioblastomas. Acta Neuropathol Commun 4:79

13. Weller M, Weber RG, Willscher E et al (2015) Molecular classification of diffuse cerebral WHO grade II/III gliomas using genome- and transcriptome-wide profiling improves stratification of prognostically distinct patient groups. Acta Neuropathol 129:679-693

14. Louis DN, von Deimling A, Cavenee WK (2016) Diffuse astrocytic and oligodendroglial tumours. In: Louis DN, Ohgaki H, Wiestler OD, Cavenee WK (eds) WHO classification of tumours of the central nervous system, revised, 4th edn. IARC, Lyon, pp 16-77

15. Wick W, Weller M, van den Bent M et al (2014) MGMT testing-the challenges for biomarker-based glioma treatment. Nat Rev Neurol 10:372-385

16. National Comprehensive Cancer Network ${ }^{\circledR}$ (2016) NCCN Clinical Practice Guidelines in Oncology (NCCN Guidelines $\left.{ }^{\circledR}\right)$. Central Nervous System Cancers. Version I. https://www.nccn.org. Accessed 25 Dec 2016

17. Malmström A, Grønberg BH, Marosi C et al (2012) Temozolomide versus standard 6-week radiotherapy versus hypofractionated radiotherapy in patients older than 60 years with glioblastoma: the Nordic randomised, phase 3 trial. Lancet Oncol 13:916-926

18. Stupp R, Mason WP, van den Bent MJ et al (2005) Radiotherapy plus concomitant and adjuvant temozolomide for glioblastoma. $\mathrm{N}$ Engl J Med 352:987-996

19. Kinoshita M, Sakai M, Arita $H$ et al (2016) Introduction of high throughput magnetic resonance T2-weighted image texture analysis for WHO grade 2 and 3 gliomas. PLoS ONE 11:e0164268

20. Okita Y, Nonaka M, Shofuda T et al (2014) (11)C-methinine uptake correlates with MGMT promoter methylation in nonenhancing gliomas. Clin Neurol Neurosurg 125:212-216

21. Uzuka T, Asano K, Sasajima T et al (2014) Treatment outcomes in glioblastoma patients aged 76 years or older: a multicenter retrospective cohort study. J Neurooncol 116:299-306

22. Louis DN, Perry A, Reifenberger G et al (2016) The 2016 World Health Organization Classification of tumors of the central nervous system: a summary. Acta Neuropathol 131:803-820

23. Arita H, Narita Y, Fukushima S et al (2013) Upregulating mutations in the TERT promoter commonly occur in adult malignant gliomas and are strongly associated with total 1p19q loss. Acta Neuropathol 126:267-276

24. Wiestler B, Claus R, Hartlieb SA et al (2013) Malignant astrocytomas of elderly patients lack favorable molecular markers: an analysis of the NOA-08 study collective. Neuro Oncol 15:1017-1026

25. Keime-Guibert F, Chinot O, Taillandier L et al (2007) Radiotherapy for glioblastoma in the elderly. N Engl J Med 356:1527-1535

26. Arvold ND, Tanguturi SK, Aizer AA et al (2015) Hypofractionated versus standard radiation therapy with or without temozolomide for older glioblastoma patients. Int J Radiat Oncol Biol Phys 92:384-389

27. Fukai J, Nishibayashi H, Uematsu Y et al (2016) Rapid regression of glioblastoma following carmustine wafer implantation: a case report. Mol Clin Oncol 5:153-157

28. Arvold ND, Cefalu M, Wang Y, Zigler C, Schrag D, Dominici F (2017) Comparative effectiveness of radiotherapy with vs. without temozolomide in older patients with glioblastoma. J Neurooncol 131:301-311

29. Franceschi E, Depenni R, Paccapelo A et al (2016) Which elderly newly diagnosed glioblastoma patients can benefit from radiotherapy and temozolomide? A PERNO prospective study. J Neurooncol 128:157-162

30. Babu R, Komisarow JM, Agarwal VJ et al (2016) Glioblastoma in the elderly: the effect of aggressive and modern therapies on survival. J Neurosurg 124(4):998-1007 


\section{Affiliations}

Takahiro Sasaki ${ }^{1,2} \cdot$ Junya Fukai ${ }^{1,2}$ (1) $\cdot$ Yoshinori Kodama ${ }^{2,3,18} \cdot$ Takanori Hirose $^{2,4} \cdot$ Yoshiko Okita $^{2,5}$. Shusuke Moriuchi ${ }^{2,6} \cdot$ Masahiro Nonaka $^{2,7} \cdot$ Naohiro Tsuyuguchi $^{2,8} \cdot$ Yuzo Terakawa $^{2,8} \cdot$ Takehiro Uda $^{2,8}$. Yusuke Tomogane $^{2,9} \cdot$ Manabu Kinoshita ${ }^{2,10} \cdot$ Namiko Nishida $^{2,11} \cdot$ Shuichi Izumoto ${ }^{2,12} \cdot$ Yoshikazu Nakajima ${ }^{2,13}$.

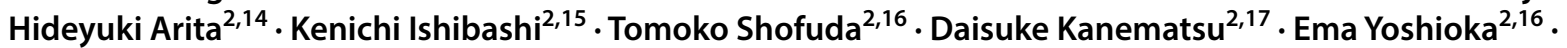
Masayuki Mano $^{2,18} \cdot$ Koji Fujita $^{1,2} \cdot$ Yuji Uematsu ${ }^{1,2} \cdot$ Naoyuki Nakao $^{1,2} \cdot$ Kanji Mori $^{2,19} \cdot$ Yonehiro Kanemura ${ }^{2,5,17}$

1 Department of Neurological Surgery, Wakayama Medical University School of Medicine, Kimiidera 811-1, Wakayama 641-0012, Japan

2 Kansai Molecular Diagnosis Network for CNS Tumors, Osaka, Japan

3 Department of Pathology and Applied Neurobiology, Graduate School of Medical Science, Kyoto Prefectural University of Medicine, Kyoto, Japan

4 Department of Diagnostic Pathology, Hyogo Cancer Center, Hyogo, Japan

5 Department of Neurosurgery, Osaka National Hospital, National Hospital Organization, Osaka, Japan

6 Department of Neurosurgery, Rinku General Medical Center, Izumisano, Osaka, Japan

7 Department of Neurosurgery, Kansai Medical University, Osaka, Japan

8 Department of Neurosurgery, Osaka City University Graduate School of Medicine, Osaka, Japan

9 Department of Neurosurgery, Hyogo College of Medicine, Nishinomiya, Hyogo, Japan

10 Department of Neurosurgery, Osaka International Cancer Institute, Osaka, Japan
11 Department of Neurosurgery, Tazuke Kofukai Foundation, Medical Research Institute, Kitano Hospital, Osaka, Japan

12 Department of Neurosurgery, Kindai University Faculty of Medicine, Osaka, Japan

13 Department of Neurosurgery, Sakai City Medical Center, Osaka, Japan

14 Department of Neurosurgery, Osaka University Graduate School of Medicine, Osaka, Japan

15 Department of Neurosurgery, Osaka City General Hospital, Osaka, Japan

16 Division of Stem Cell Research, Department of Biomedical Research and Innovation, Institute for Clinical Research, Osaka National Hospital, National Hospital Organization, Osaka, Japan

17 Division of Regenerative Medicine, Department of Biomedical Research and Innovation, Institute for Clinical Research, Osaka National Hospital, National Hospital Organization, Osaka, Japan

18 Department of Pathology, Osaka National Hospital, National Hospital Organization, Osaka, Japan

19 Department of Neurosurgery, Kansai Rosai Hospital, Hyogo, Japan 